\title{
Transoral Laser Microsurgery for Malignancies of the Upper Aerodigestive Tract
}

\section{Jason Durel, 2Jacques Gaudet, ${ }^{3}$ Melda Kunduk, ${ }^{4}$ Andrew J McWhorter}

${ }^{1}$ Resident Physician, Department of Otolaryngology-Head and Neck Surgery, Louisiana State University Health Sciences Center, New Orleans, Louisiana

${ }^{2}$ Resident Physician, Department of Otolaryngology-Head and Neck Surgery, Louisiana State University Health Sciences Center, New Orleans, Louisiana

${ }^{3}$ Assistant Professor, Department of Otolaryngology-Head and Neck Surgery, Louisiana State University Health Science Center, New Orleans, Louisiana

${ }^{4}$ Assistant Professor, Director, Department of Otolaryngology-Head and Neck Surgery, Louisiana State University Health Sciences Center, New Orleans, Louisiana

Correspondence: Andrew J McWhorter, Assistant Professor, Director, Department of Otolaryngology-Head and Neck Surgery Louisiana State University Health Sciences Center, Our Lady of the Lake Voice Center, 7777 Hennessy Boulevard, Suite 408 Baton Rouge, Louisiana 70808, e-mail: amcwhort@ololrmc.com

\section{Abstract}

Transoral laser microsurgery (TLM) is an important option in the treatment plan for tumors of the oral cavity, oropharynx, larynx, and hypopharynx. For properly selected patients, TLM provides equivalent oncologic outcomes when compared to traditional therapies, while improving the functional aspects of postoperative speech, voice and swallowing.

Keywords: Transoral laser microsurgery, larynx, oral cavity, oropharynx, hypopharynx.

\section{INTRODUCTION}

\section{History}

The practice of transoral removal of laryngeal neoplasms predated the development of illuminated endoscopes specifically designed for that purpose. In 1852, Horace Green reported use of a bent tongue spatula with sunlight illumination to remove a ball-valving fibroepithelial polyp in a child. ${ }^{1}$ Development of indirect mirror-guided approaches to the larynx led to the first report of transoral resection of a laryngeal cancer by Bernhard Fraenkel in 1886. Kirstein later developed endoscopes designed specifically to directly expose the larynx in the 1890's. ${ }^{2}$

Transoral endoscopic management of laryngeal cancer has continued to evolve with the introduction of suspension laryngoscopy in 1920 and microscopic visualization of the larynx in $1960 .{ }^{3,4}$ Strong and Jako first described transoral laser microsurgery (TLM) for laryngeal malignancies in $1972,{ }^{5}$ accomplishing their resections with use of the carbon dioxide laser. Complications from early laser surgery and poor oncologic outcomes prevented laser resection from gaining prominence in the United States while advances in radiation oncology and chemotherapy progressed resulting in a notable increase in the nonsurgical treatment of laryngeal cancer through the 1980's and 1990's. ${ }^{6}$

During this same period, however, a paradigm shift was occurring in Germany where surgeons expanded on Strong and Jako's techniques to address even larger tumors. ${ }^{7,8}$ Improved instrumentation resulted in improved access and visualization (Fig. 1). Refinement of carbon dioxide laser technology including laser-pulsing and computer-generated patterns improved the surgeon's ability to accurately resect these lesions under microscopic visualization. Success with laryngeal tumors led them to tackle lesions of the oropharynx and hypopharynx with the same techniques. This work, although initially discounted, through careful documentation and perseverance has gained widespread acceptance from surgeons across the globe.

\section{Technique and Concepts}

Transoral laser microsurgery (TLM) for resection of laryngeal malignancies challenges traditional oncologic approaches. The limits of visualization through the 


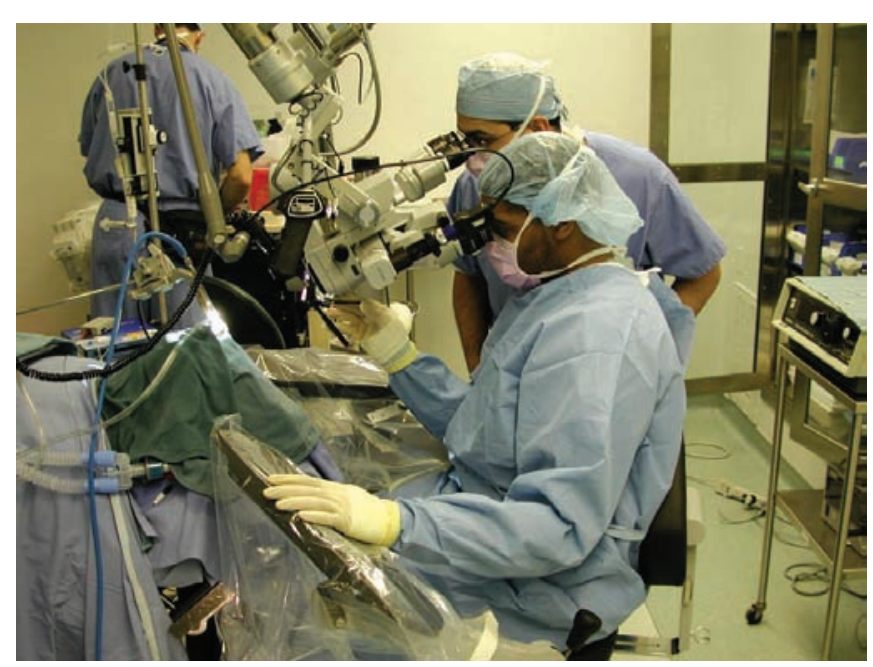

FIGURE 1: Standard operating room set up for transoral laser microsurgery with $\mathrm{CO}_{2}$ laser, operating chair, microscope, suspended laryngoscope, plume evacuation and laser precautions in place for the patient and operating room personnel

laryngoscope dictate the size of the tumor that can be managed in an en bloc resection. The first tenet of TLM violates Halsted's primary principle of not violating the tumor for two reasons. First, transection with piecemeal removal of tumor allows for the resection of larger tumors through the laryngoscope. Second, transection demonstrates depth of penetration of the tumor and allows for improved visualization of margins during extirpation. This concept has been upheld in multiple series with equivalent or better local control rates compared to traditional treatment modalities. The concept of piecemeal removal should not be misinterpreted as a less radical resection. Tumor volumes and margins should be equivalent to traditional open approaches. Similarly, tumors are not vaporized but rather are excised for pathologic evaluation.

Procedures are performed similarly to traditional direct suspension microlaryngoscopy. Tracheotomies are avoided whenever possible to promote postoperative rehabilitation, and ventilation is performed via small laser protected endotracheal tubes. Specialized nonreflective laryngoscopes are placed in suspension with visualization through the microscope with an attached micromanipulator for $\mathrm{CO}_{2}$ laser delivery. Appropriate protective measures are ensured for laser safety (Fig. 2).

Traditional concepts of conservation laryngeal surgery are observed with maintenance of at least one laryngeal valve (true vocal cords, false vocal cords, or epiglotticaryepiglottic valve) for adequate airway protection and voice generation. One functional crico-arytenoid unit at a minimum must be able to be conserved. Functional status

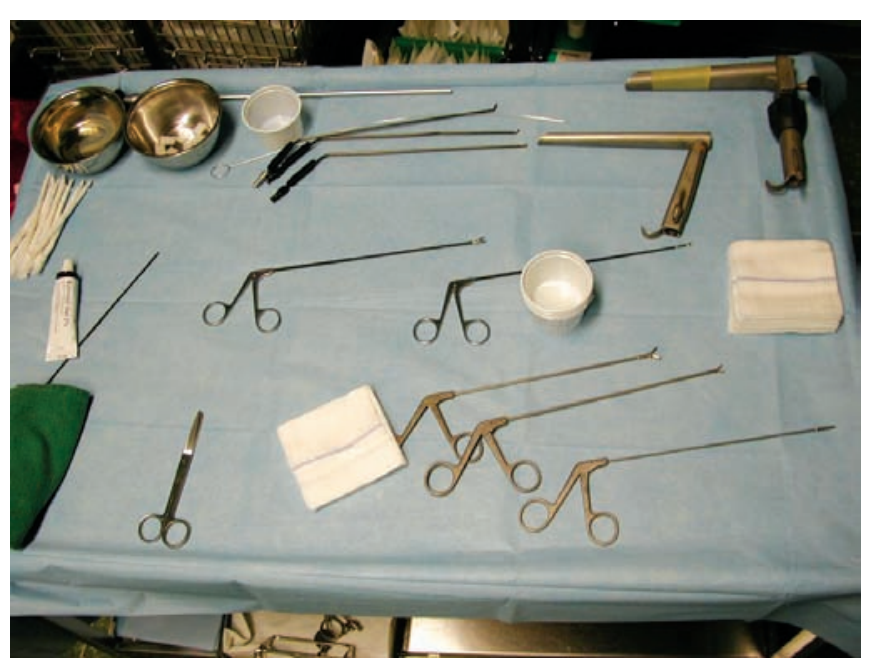

FIGURE 2: Standard back table set up with assorted laryngoscopes and microlaryngeal equipment for endoscopic resection

of the patient and medical co-morbidities are similarly important. Adequate exposure for access for resection is also necessary. Extralaryngeal spread with involvement of the great vessels or need for reconstruction are contraindications. The experience and skill of the surgeon with regards to both laryngoscopy and TLM techniques are paramount in attaining optimal outcomes. Proper equipment is also a prerequisite.

There are no absolute rules used as indications for a particular resection. Each resection is tailored to the patient with the concept of "following the tumor". The tumor is transected as aforementioned, dissection proceeds around the tumor with appropriate margins taken. The chromophore for the carbon dioxide laser is water, and cells contacted by the laser will have their water content heated to the point that they will rupture. Due to differing amounts of water in tumor tissue as opposed to healthy tissue, laser-tissue interactions and tissue carbonization are used as a marker of tumor extent. ${ }^{9}$ Vocal fold margins are felt to be adequate at $1-3 \mathrm{~mm}$ with larger margins of $5-10 \mathrm{~mm}$ taken in the supraglottis and hypopharynx. In previously irradiated fields, larger margins of resection should be utilized. It is very important to maintain proper orientation of the specimens and work closely with the pathologist. Margins of resection can be difficult to interpret if there is not good coordination of the specimen evaluation process (Fig. 3). Reconstruction is not typically performed, and healing is by secondary intention. Granulation tissue forms, followed by contraction and remucosalization with the contracture process helping to eliminate dead space. 


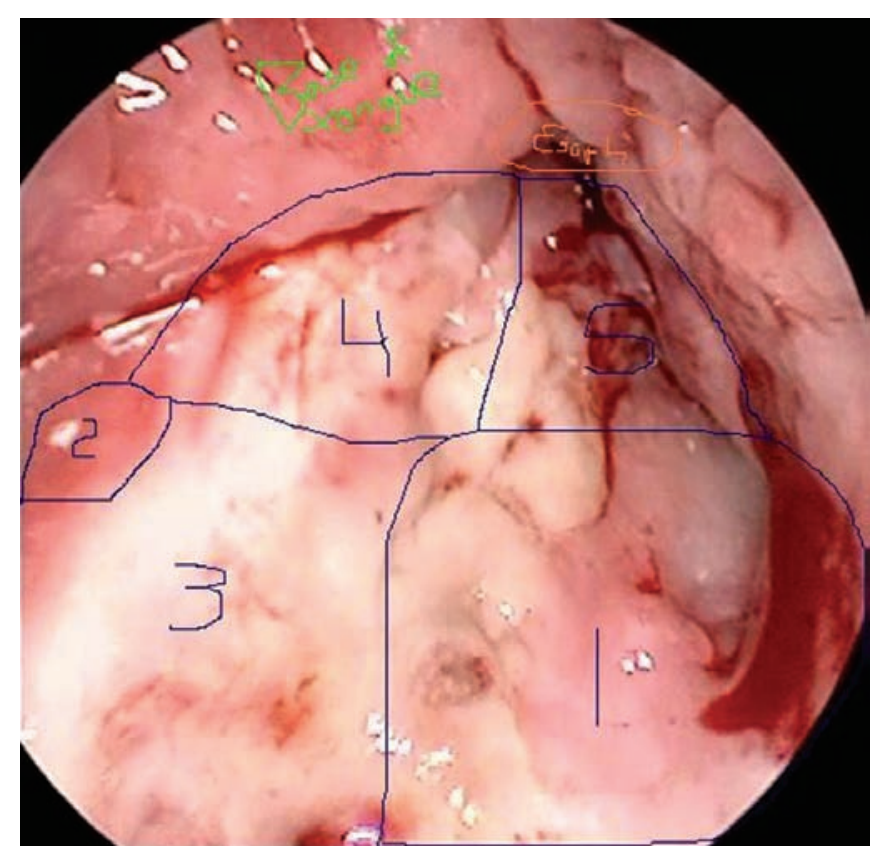

FIGURE 3: Photo marked as a tumor map of a posterior pharyngeal wall carcinoma for communication of specimen margins and orientation with the pathologist

\section{ONCOLOGIC OUTCOMES}

\section{Larynx}

\section{Glottis}

Early: TLM has been well-studied in the treatment of early glottic malignancies, and its utility for these lesions is wellestablished (Figs 4A and B). In a recent retrospective analysis conducted by Thurnher et al, TLM (and laryngofissure) showed significant advantages in terms of disease free survival, locoregional recurrence rate, time to locoregional recurrence, and laryngeal preservation when compared to radiation therapy. ${ }^{10}$ (Table 1) Multiple other studies also found TLM to be similarly efficacious in management of these malignancies. ${ }^{8,9,11}$

Controversy exits on the importance of anterior commissure involvement in the applicability of TLM. Some reports hold that due to the difficulty in visualization or microinvasion through Broyles' ligament, anterior
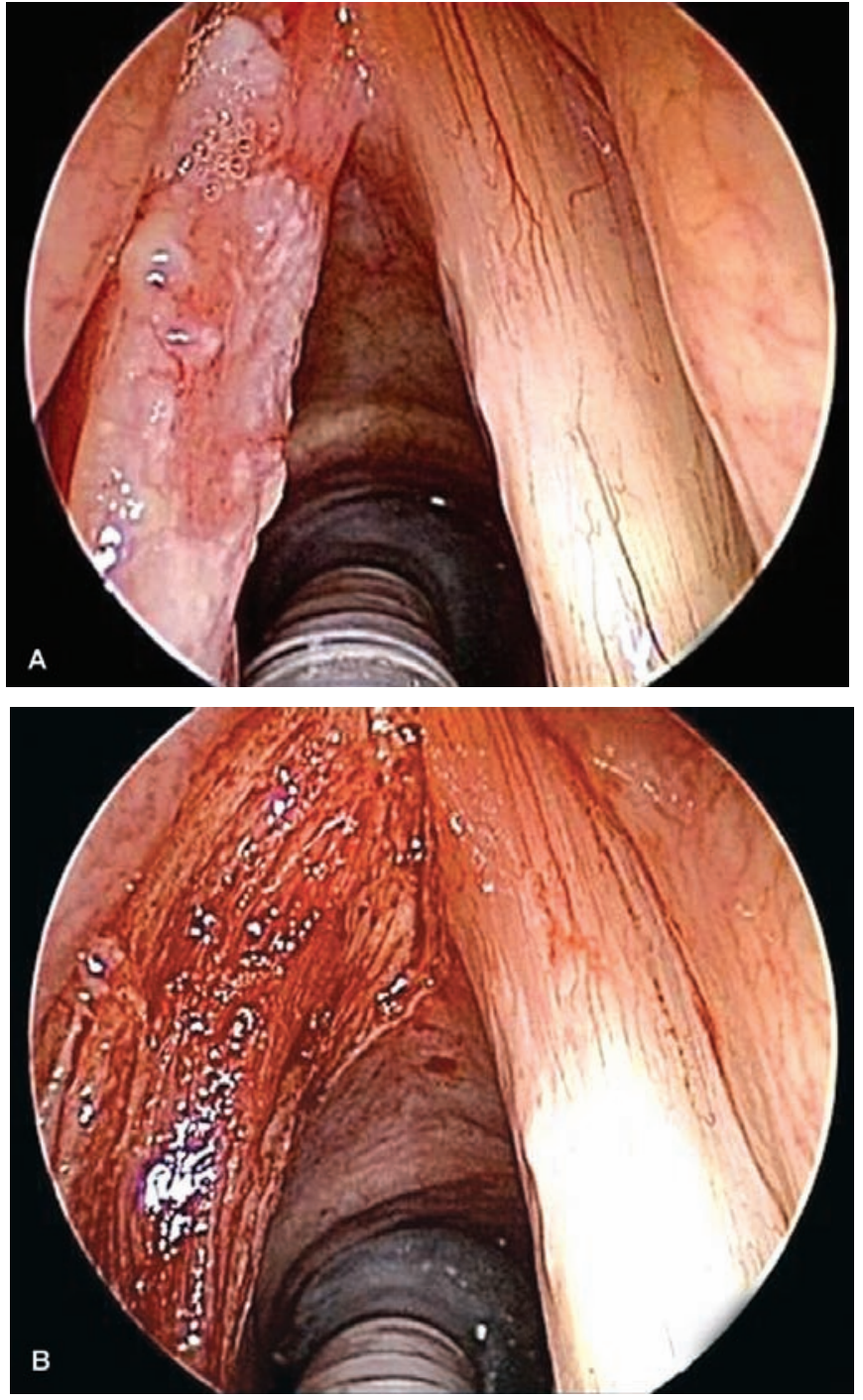

FIGURES 4A and B: (A) T1NOM0 squamous cell carcinoma left true vocal fold intraoperative photo prior to resection with laser shielded endotracheal tube in place. (B) status post TLM resection of tumor

commissure disease is difficult to manage transorally. The published data on anterior commissure involvement is inconsistent, however, some series have published excellent results. ${ }^{12,13}$

Ansarin et al noted that positive margin status significantly increases the risk of local recurrence when using TLM to address laryngeal tumors. ${ }^{14}$

TABLE 1: Oncologic outcomes from Thurnher et al ${ }^{10}$

\begin{tabular}{lcccccc}
\hline & \multicolumn{3}{c}{ Disease specific survival } & Locoregional & \multicolumn{2}{c}{$\begin{array}{c}\text { Time to } \\
\text { recurrence }\end{array}$} \\
& 5 years & 10 years & 15 years & recurrence & preal \\
\hline TLM $(\mathrm{n}=81)$ & $100 \%$ & $100 \%$ & $100 \%$ & $10 \%$ & 58.5 months & $100.0 \%$ \\
Laryngofissure $(\mathrm{n}=148)$ & $100 \%$ & $98 \%$ & $98 \%$ & $13 \%$ & 43 months & $91.9 \%$ \\
XRT ( $=108)$ & $96 \%$ & $92 \%$ & $91 \%$ & $30 \%$ & 26 months & $84.3 \%$ \\
\hline
\end{tabular}



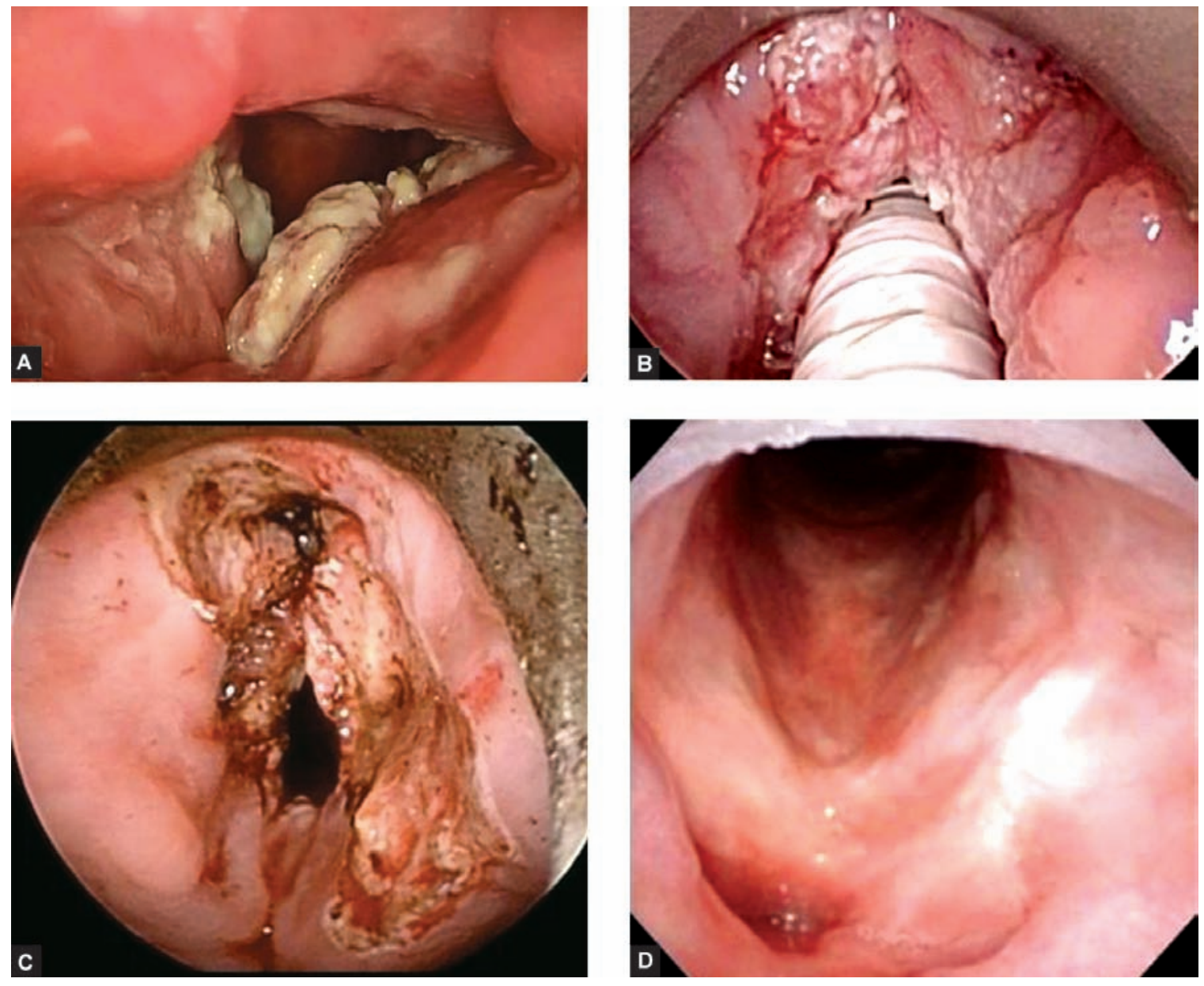

FIGURES 5A to D: (A) rT2N0M0 squamous cell carcinoma recurrence following radiation therapy in a patient with a history of recurrent respiratory papilloma (preoperative in office 70 degree rigid scope view). (B) intraoperative photo of same tumor with laser shielded endotracheal tube in position. (C) following resection of tumor from false and true vocal folds and right arytenoid. (D) 1 year follow-up flexible laryngoscopy view with scar of left true and false vocal fold and no evidence of disease

\section{Advanced}

TLM has a growing role in the management of advanced laryngeal malignancies. Hinni et al reviewed 117 patients with stage III and IV glottic and supraglottic disease managed with TLM and demonstrated 2 and 5 year overall survival rates of $75 \%$ and $55 \%$ respectively with laryngeal preservation of $86 \%$ at 5 years. ${ }^{15}$ These numbers correlate favorably with outcomes of nonsurgical treatment modalities in the landmark RTOG study. In their conclusion, the authors of this article state that TLM "is an effective treatment for advanced laryngeal cancer" and challenge the assertion of the RTOG study that "radiotherapy with concurrent cisplatin should be considered standard of care for patients desiring laryngeal preservation". ${ }^{15,16}$

\section{Supraglottis}

Supraglottic lesions have a generally poorer prognosis than their glottic counterparts secondary to the inherent trend towards later presentation and higher rates of metastatic cervical disease (Figs 5A to 6F).

Ambrosch et al reviewed $48 \mathrm{~T} 1$ and $\mathrm{T} 2$ supraglottic lesions treated with TLM and reported 5 years local control rates of with first treatment of $100 \%$ for T1 and $89 \%$ for $\mathrm{T} 2$ lesions. ${ }^{17}$ Rodrigo et al reviewed the results of TLM for in $215 \mathrm{~T} 1 / \mathrm{T} 2$ supraglottic lesions and found an approximately $11 \%$ incidence of local recurrence. Results for patients with T3 lesions were slightly worse with 13 local recurrences in 70 patients (18.5\%). Rodrigo concludes that these recurrence rates are comparable to those for open 

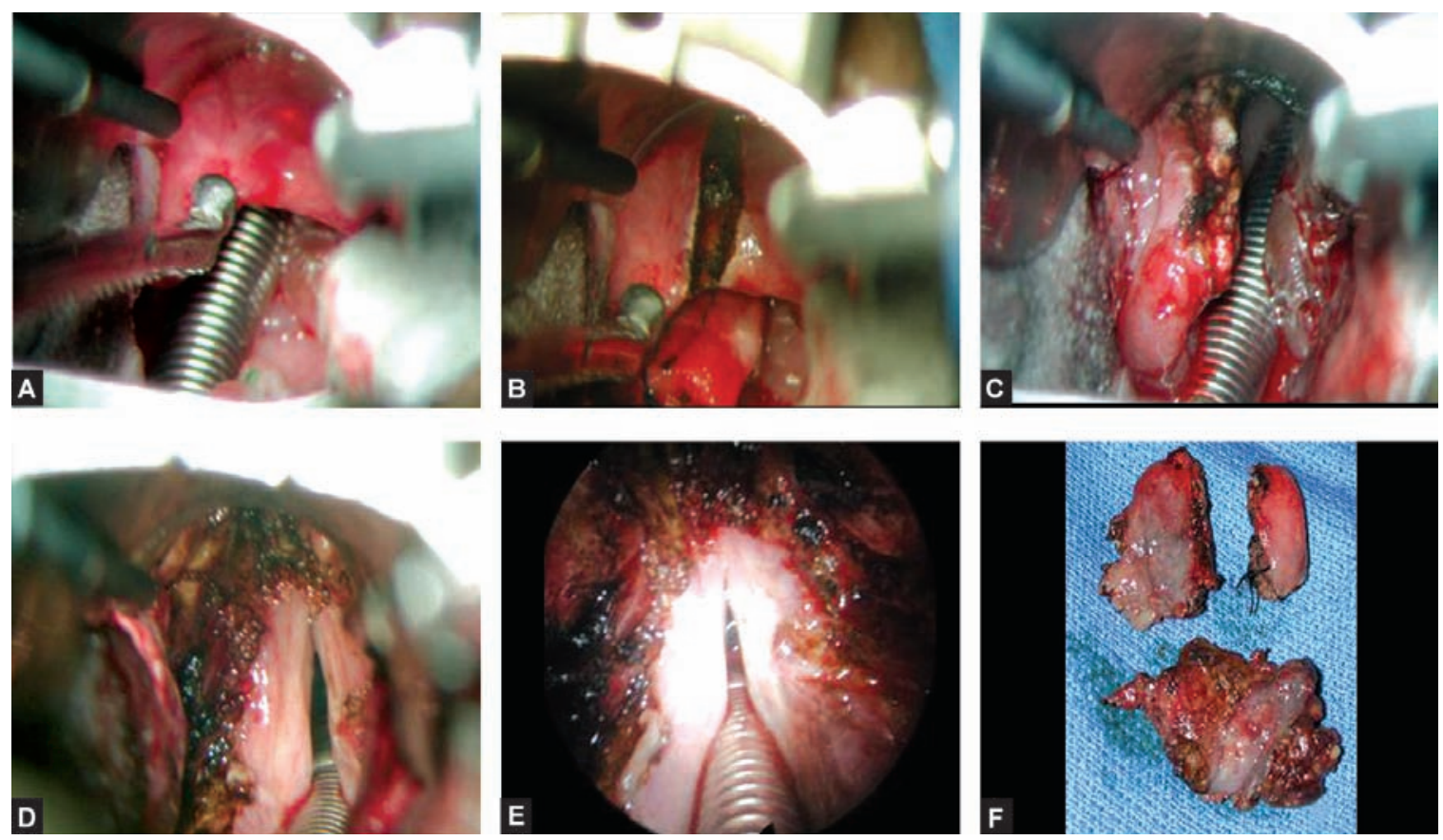

FIGURES 6A to F: (A) Photo of lingual surface of epiglottis with aiming beam of $\mathrm{CO}_{2}$ laser visible and up biting cup through bivalve laryngoscope with laser shielded endotracheal tube. (B) $\mathrm{CO}_{2}$ laser splitting of epiglottis at beginning of transoral endoscopic supraglottic laryngectomy for T2N0M0 squamous cell carcinoma. (C) right half of epiglottis removed with visible remaining tumor and left hemiepiglottis and laser shielded endotracheal tube. (D) microscope view following TLM supraglottic laryngectomy with visible preserved true vocal folds. (E) zero degree telescope photo of TLM supraglottic laryngectomy. (F) oriented 3 piece pathologic specimen of supraglottic tumor resection

surgery and primary radiation therapy, but warns that TLM should be used cautiously in T3 supraglottic tumors, as complete resection may be difficult. ${ }^{18}$

\section{Hypopharynx}

Both endoscopic examination and computed tomographic imaging are critical in assessing hypopharyngeal tumors, as endoscopy frequently underestimates the tumor size. Such masses routinely invade the paraglottic or pre-epiglottic spaces, soft tissues of the neck, or the thyroid cartilage without any evidence of such invasion on routine endoscopy.

The prognosis for patients with hypopharyngeal cancer is generally poor regardless of the therapeutic intervention. Oncologic outcomes for patients treated with TLM are no exception. TLM does, however, offer similar oncologic outcomes to other modalities with a greater rate of laryngeal preservation and function (Figs 7A and B). Vilaseca et al reviewed 28 patients with hypopharyngeal cancer treated with TLM. Tumor staging was as follows: $\mathrm{T} 1=2, \mathrm{~T} 2=$ $16, \mathrm{~T} 3=9, \mathrm{~T} 4=1$. Four year overall and disease-specific survival was $43.4 \%$ and $59.4 \%$ respectively. Outcomes were essentially equivalent to those with other modalities, however, $78 \%$ of TLM patients maintained laryngeal function as opposed to $8 \%$ of the patients treated with other modalities. ${ }^{19}$ Martin et al reviewed 172 patients with hypopharyngeal lesions treated with TLM with or without adjuvant chemoradiation..$^{20} 85 \%$ of their patients had stage III and IVa lesions. Five year recurrence-free survival was $73 \%$ for Stage I and II, 59\% for Stage III, and 47\% for Stage IVa. Again, this data compares favorably to that of other modalities. The authors thus concluded that their results "prove that selected hypopharyngeal cancers of all categories T1-T4 can be treated successfully by TLM, without comprising the oncologic outcome and with a high rate of organ and function preservation". ${ }^{20}$

\section{Oropharynx and Oral Cavity}

The role of TLM in management of oral cavity and oropharyngeal tumors is less established. Traditionally, single modality therapy with surgery or radiation has been used for T1 or T2 lesions. For advanced (T3 and T4) lesions, dual modality treatment with either chemoradiation or surgery with postoperative radiation is advocated. It has been suggested that TLM may offer an advantage in its ability to offer greater exposure to otherwise inaccessible lesions of the oropharynx. ${ }^{21}$

Eckel et al investigated the results of TLM with staged neck dissections in 117 patients with oral cavity or 

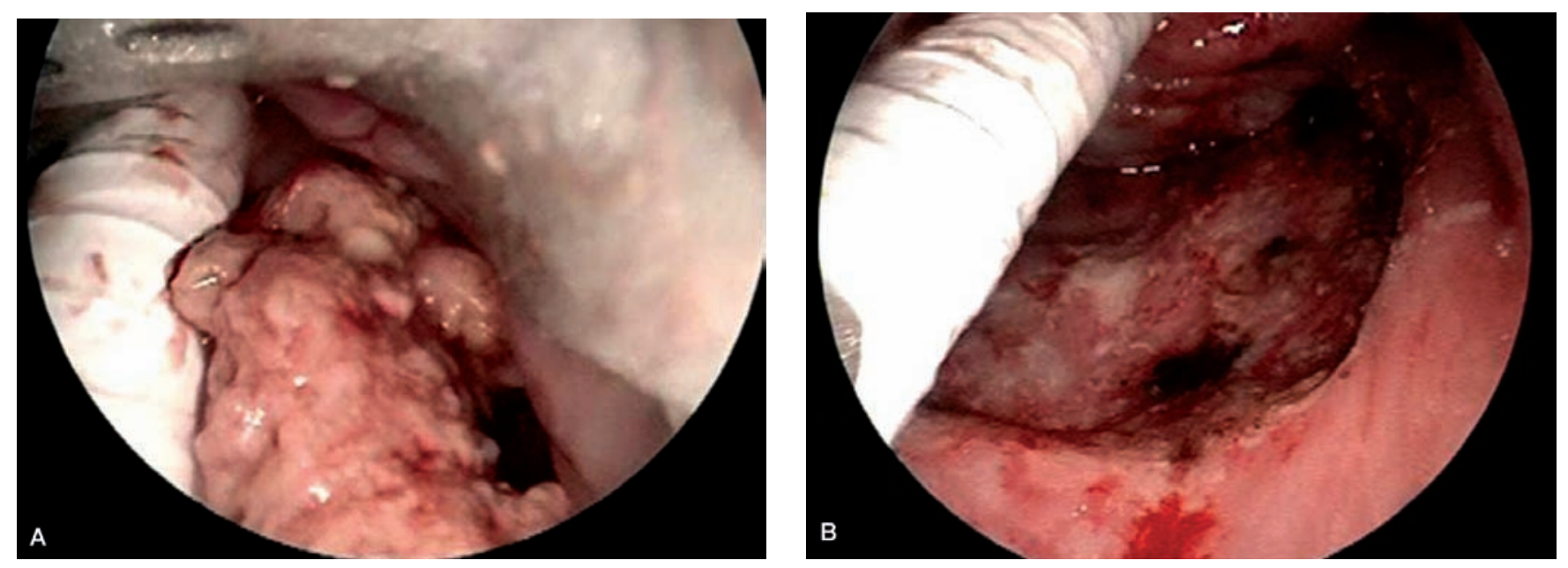

FIGURES 7A and B: (A) T2NOM0 squamous cell carcinoma of posterior pharyngeal wall with bivalve scope at top and laser shielded endotracheal tube left. (B) status post TLM resection of tumor with posterior larynx and laser shielded endotracheal tube visible

oropharyngeal squamous cell carcinoma. Oncologic outcomes in this prospective trial were in accordance with previously reported results from other modalities of treatment (Table 2).22

Steiner et al reviewed the use of TLM specifically for tongue base primaries in 48 patients. $94 \%$ of these patients had stage III/IVa tumors. Staged neck dissection and postoperative radiotherapy with or without chemotherapy were administered when appropriate. No patients with T1/T2 disease had local recurrence, but $20 \%$ of patients with T3/T4 disease locally recurred. ${ }^{23}$ Five year overall local control rates for tongue base tumors treated with primary radiotherapy are between $44 \%$ and $78 \% .^{24-26}$

\section{Neck Management}

When Crile initially demonstrated the survival advantage of en bloc resection of the primary tumor with synchronous

TABLE 2: Oncologic results from Eckel et al review of TLM for oral cavity and oropharyngeal tumors ${ }^{22}$

\begin{tabular}{lc}
\hline & 5 years DSS \\
\hline Oral cavity $(\mathbf{n}=\mathbf{6 4})$ & \\
Stage I/II & $81 \%$ \\
Stage III & $73 \%$ \\
Oropharynx $(\mathbf{n}=\mathbf{5 3})$ & \\
Stage I/II & $83 \%$ \\
Stage III & $65 \%$ \\
Combined OC/OP & \\
Stage IV & $21 \%$ \\
\hline
\end{tabular}

DSS: Disease specific survival, OC: Oral cavity, OP: Oropharynx. lymphadenectomy, TLM did not exist. TLM violates the concept of en bloc resection and has thus led to a discussion on the appropriate management of the neck in these cases. Should these patients undergo discontinuous removal of at risk nodes at time of surgery, or should the removal of such nodes occur as a staged procedure to allow for metastases in transit to reach their respective nodal basins? This topic is still debatable.

Leemans et al reviewed 61 patients with oral malignancies and showed improved overall survival with continuous, synchronous removal of the primary and neck dissection (80.2\%) as compared to TLM and discontinuous, synchronous neck dissection $(67.1 \%) .{ }^{27}$ Tesseroli et al retrospectively reviewed neck management in 193 patients with oral cavity carcinoma. The study had three groups: (1) synchronous continuous excision of the primary lesion and neck dissection; (2) resection of primary lesion with synchronous discontinuous neck dissection; and (3) resection of the primary lesion with delayed discontinuous neck dissection. In the three groups, there was no significant difference observed in disease-free survival or cancerspecific survival in any group. ${ }^{28}$

\section{Salvage TLM Surgery}

Recurrent or persistent disease in a previously irradiated field presents specific treatment issues for the surgeon. Radiation-induced changes to surrounding tissue often make it difficult to histopathologically determine the extent of recurrent or persistent lesions. ${ }^{29}$ Margins should therefore be greater in patients undergoing TLM for radiation failure. ${ }^{30}$ 
Steiner et al investigated the use of TLM as surgical salvage in 34 patients who had recurrent carcinoma of the glottis after radiation therapy, and showed good oncologic outcomes. ${ }^{30}$ For rT1/rT2 disease, $71.4 \%$ of patients were disease-free at a median follow-up of three years. For rT3/ rT4 disease, $69 \%$ of patients were disease-free at the same follow-up.

Grant et al reported on 114 patients who were treated with TLM for recurrent disease after surgery and/or radiation with curative intent for primary lesions of the larynx or pharynx. In patients treated with prior radiotherapy or chemoradiotherapy, two year locoregional control rates were $88 \%$ for laryngeal tumors, $80 \%$ for hypopharyngeal tumors, and $55 \%$ for oropharyngeal tumors. ${ }^{31}$ These oncologic outcomes are in line with those achieved through any modality when treating recurrent disease.

\section{FUNCTIONAL OUTCOMES}

\section{General Functional Advantages of TLM}

In most TLM cases, the larynx maintains its mobility and elevation by avoidance of tracheotomy and preservation of the strap muscles. This enables the larynx to assume its normal path in deglutition and provides the natural protection of the tongue base. The avoidance of a tracheotomy in $95 \%$ or more TLM cases ${ }^{32}$ improves swallowing outcomes and patient satisfaction while decreasing length of stay and healthcare costs. Laryngeal sensation is also often better preserved in the endoscopic procedures allowing better bolus localization and control. ${ }^{33}$ Multiple series have demonstrated a faster return to swallowing and decreased use of feeding tubes when compared to equivalent open procedures. Decreased rates of pharyngocutaneous fistula and aspiration pneumonia have also been documented. ${ }^{18}$

\section{Preoperative Planning}

An optimal protocol for rehabilitation starts with realistic expectations and frank discussion of expected postoperative function, which can decrease anxiety and frustration during the rehabilitation process. Prophylactic gastrostomy tube placement rather than perioperative nasogastric tube placement is beneficial if prolonged dysphagia is expected. The ability to maintain hydration and nutritional support via the gastrostomy tube decreases the anxiety associated with inability to swallow and eliminates the urgency of safe swallowing, thus allowing a more beneficial therapy experience. A thorough preoperative assessment of swallow, voice, and speech function is also critical. In addition to a detailed history and physical, this may include (but is certainly not limited to) radiologic evaluation of the swallowing mechanism and endoscopic evaluation of swallowing with or without sensory testing (FEES or FEESST). ${ }^{34,35}$

The success of voice, speech, swallowing, and respiratory rehabilitation following transoral laser surgery(TLM) requires a multidisciplinary approach. Comprehensive evaluation and teamwork between the surgeon, speech pathologist, radiologist, dietitian, medical and radiation oncologists, prosthodontist, and social worker are critical.

\section{SITE-SPECIFIC FUNCTIONAL OUTCOMES AND THERAPEUTIC IMPLICATIONS}

\section{Overview}

Patients with voice and swallowing difficulties may experience tremendous frustration and loss of social, cultural and personal satisfaction. Difficulty with speech and swallowing may also contribute to loss of income and depression.

The goals of swallowing rehabilitation are to minimize aspiration risk and resume regular oral diet. The initiation of post surgical rehabilitation should begin within 2-3 days of surgery, focusing on counseling and informative sessions on functional effects of the surgery. ${ }^{36}$ The timing of aggressive swallowing rehabilitation will depend on the extent of the surgery and healing of the surgical bed, but usually starts within one to two weeks of surgery. ${ }^{36}$

\section{Oral Cavity}

The important factors determining postoperative swallowing function following TLM for oral cavity cancers are the site and amount of resected tissue. ${ }^{11}$ In addition to structural deficiencies, the loss of sensation and motor function can play a critical role in postoperative dysphagia. Loss of sensation may interfere with bolus formation and impede the timely delivery of the bolus to the pharynx, may result in aspiration prior to swallow due to an unprotected airway. Decreased oral sensation and motor function and structural competence may also result in host of other mechanical issues, ranging from difficulty with bolus management, to impaired laryngeal elevation, nasal regurgitation and more significant dysphagia. ${ }^{36-38}$ The intelligibility of speech may also be affected due to the change in tongue motion and resonance issues. 
Behavioral therapy, dietary modification and use of intraoral prosthetics techniques are commonly employed for these patients. Tongue and jaw range motion exercises, ${ }^{36}$ bolus manipulation exercises, tongue base retraction exercises, ${ }^{39}$ swallowing maneuvers ${ }^{36,38}$ (super-supraglottic swallow, effortful swallow, Mendelssohn's maneuver, and Shaker's exercises ${ }^{40}$ ) can be used to offset stricture or scar formation and promote efficient and safe swallowing. This exercise regimen becomes especially important if radiation is in the treatment plan, as this is an independent predictor of poorer functional outcome. ${ }^{41-44}$ Intraoral prosthetics can help with speech and swallowing difficulties due to palatal defects and reduced tongue mobility. Treatment of defects in adynamic structures appears to result in better functional outcome than defects in dynamic structures. Manipulating food consistency can help with propulsion and bolus flow since thicker consistencies will be especially hard to manipulate for the patient with a tongue defect. However, it is very important to ensure that the patient is capable of protecting their airway prior to starting diet modification.

\section{Oropharynx and Hypopharynx}

Compromised sensory and motor function of the structures in these areas may interfere with the sensation of bolus arrival into pharynx, bolus flow through the pharynx, and bolus entry into esophagus. The tongue base is critical for the initiation of swallow and transition of the bolus from pharynx into esophagus. Pharyngeal peristalsis is vital for stripping of the bolus from pharynx before the airway opens. The piriform sinuses are essential for directing the bolus into esophagus. Structural changes in these organs may lead to aspiration due to lack of sensation and residue in vallecula, posterior pharyngeal wall, and piriform sinuses before and after swallow. Base of tongue and hypopharyngeal tumors treated with TLM show better swallowing outcomes compared those treated with conventional treatment strategies, likely due to improved pharyngeal sensation following TLM as opposed to traditional surgery. ${ }^{33,45}$ Voice effects following surgery for oropharyngeal and hypopharyngeal tumors are usually less significant than the dysphagia.

Early initiation of swallowing rehabilitation tailored to address with each patient's structural deficits will ensure the best functional outcome possible. Exercise to improve function of tongue base (such as Masako exercises), ${ }^{39}$ pharyngeal wall (pharyngeal squeeze), ${ }^{46}$ upper esophageal sphincter (Shaker's exercise) ${ }^{40}$ and change in the consistency of food can improve the efficiency of swallowing.

\section{Larynx}

\section{Glottis}

Treatment of early glottic cancers with TLM primarily affects voice with minimal effect on swallowing function. Resection of smaller tumors usually results in very good functional outcome after the postoperative healing period. More extensive vocal muscle resection will cause a permanent alteration in voice quality. Multiple studies have demonstrated that extended cordectomy involving the entire vocal fold and/or anterior commissure demonstrated worse outcome than more limited procedures in addition to permanent voice change. ${ }^{47}$ Surgical strategies to maintain good voice include avoidance of webbing at the anterior commissure and maintaining one undisturbed fold for vibration when possible. Mitomycin $\mathrm{C}$ has been shown to reduce scarring in the anterior larynx and contribute to improved voice outcomes. ${ }^{48}$ Zeitels has reported the early use of medialization techniques to improve glottic closure after resection and significantly enhance voice quality. ${ }^{49}$ Compensatory strategies such as employing the false vocal fold as source of vibration will depend on remaining glottic structure and function following TLM.

Assuming the supraglottis is functioning, postoperative dysphagia is not a major issue. Intermittent aspiration of liquids during the early stage of recovery is typical due to the compromise in true vocal folds' sphincteric function in airway protection. Rehabilitation of these patients involve patient education on the swallowing mechanism and maybe temporarily use of swallowing maneuvers such as supraglottic swallow especially during the liquid swallow. These patients tend to have good long-term swallowing function.

\section{Supraglottis}

TLM for supraglottic tumors generally results in more significant dysphagia than for TLM for glottic tumors. Conversely, the vocal outcomes are improved for this group of tumors. ${ }^{50,51}$ With regards to the dysphagia, the extent of the excision will determine severity of the swallowing difficulties. Two of the three laryngeal valves (false vocal folds and epiglottis-aryepiglottic-arytenoids) involved in airway protection are located in the supraglottis. Therefore, the patient must learn to use the true vocal folds efficiently 
to protect his/her airway. Swallowing rehabilitation following supraglottic TLM surgery will involve combination of behavioral therapy and initial diet modification to initiate safe and efficient oral feeding. The goals are: (1) airway protection during the swallow; (2) determining a consistency that patient can eat safely and meet their nutritional needs by oral feeding only; and (3) advancing toward a more regular diet. Safe liquid intake is usually the last objective achieved due to the fast flow of liquids, absent laryngeal valves, and initial lack of coordination of the remaining swallowing mechanism.

\section{Summary of Functional Outcomes}

Treatment of malignancies of the oral cavity, oropharynx, hypopharynx or larynx, depending of the location, size and extent of the tumor can have permanent or transient effect on speech, voice, and swallowing function. TLM has been shown to be advantageous in terms of many of these functional outcomes. The successful rehabilitation for the return of swallowing speech and voice relies on accurate preoperative assessment and counseling and early initiation of individually tailored, aggressive treatment protocols for these deficits.

\section{COMPLICATIONS}

Steiner et al performed a retrospective review of TLM complications reviewing 1528 cases. ${ }^{32}$ Postoperative bleeding was the most commonly seen complication. For patients with glottic lesions, the rate of postoperative bleeding was 0.6-3.3\%. Other complications seen after TLM for glottic tumors were "synechia requiring treatment" and laryngeal edema (0-3.3\%). As expected, complication rates increased with tumor size. Of note, the T3 group had a rate of temporary tracheostomy of $3.2 \%$, while no patients in lower staged groups required surgical airway intervention. Other complications in this group included stenosis, pneumonia, cutaneous emphysema and perichondritis.

The most commonly observed complication for supraglottic lesions was endolaryngeal bleeding (6.0-9.8\%). Postoperative pneumonia was the second most common complication (2.4-5.3\%). Nine patients required temporary tracheostomy and two patients required permanent tracheostomy. Other complications in this group included laryngeal edema and laryngeal stenosis.

In patients treated for hypopharyngeal, oropharyngeal, and oral cavity lesions, postoperative bleeding occurred in $5.0-10.7 \%$ of cases. Other rarely encountered complications included fistula formation, hypoglossal nerve injury, nasal regurgitation, stenosis, and trismus. ${ }^{32}$

Preuss et al reviewed complications in a retrospective, multi-institutional review of 275 patients who underwent TLM for laryngeal malignancies. ${ }^{52}$ Intraoperative complications included significant bleeding (1.8\%), tracheostomy (1.8\%), dental injury (0.7\%), and unexpected difficult resection (1.1\%). The most common postoperative complication encountered was dysphagia/aspiration (6.2\%). Other postoperative complications included bleeding (2.9\%), dyspnea $(2.5 \%)$, and infection $(1.8 \%)$. Eleven patients had postoperative complications not specified in the paper. Late complications cited were laryngeal synechiae (8.4\%), dysphagia (3.3\%), hoarseness (3.3\%), dyspnea (1.1\%), and fistula formation $(0.7 \%)$.

\section{CONCLUSION}

Head and neck malignancies require a multidisciplinary approach, with radiation, chemotherapy, open surgery, and TLM all playing important roles in achieving optimal oncologic and functional outcomes. TLM is an important option in the treatment plan for tumors of the oral cavity, oropharynx, larynx, and hypopharynx. For properly selected patients, TLM provides equivalent oncologic outcomes when compared to traditional therapies, while improving the functional aspects of postoperative speech, voice and swallowing. In our current environment of cost-conscious medical practice, TLM's advantages of decreased hospital stay and overall decreased treatment costs may become even more important. As technology continues to advance and more surgeons gain experience with TLM, the spectrum of diseases treated is likely to continue to expand. Moreover, as we gain experience with treating these tumors with TLM, the presently good functional outcomes achieved with this modality are likely to improve even more.

\section{REFERENCES}

1. Zeitels S. Chapter 1 "History". Atlas of phonomicrosurgey and other endolaryngeal procedures for benign and malignant disease. San Diego: Singular/Thompson Learning; 2001.

2. Zeitels S, Dailey S, Burns J. Technique of en bloc laser endoscopic frontolateral laryngectomy for glottic cancer. Laryngoscope 2004;114:175-80.

3. Scalco A, Shipman W, Tabb H. Microscopic suspension laryngoscopy. Annals of Otology, Rhinology, and Laryngology 1960;69:1134-38.

4. Lynch R. Intrinsic carcinoma of the larynx with a second report of the cases operated on by suspension and dissection. 
Transactions of the American Laryngologic Association 1920; 42:119-26.

5. Strong M, Jako G. Laser surgery in the larynx: Early clinical experience with continuous $\mathrm{CO}_{2}$ laser. Annals of Otology, Rhinology, and Laryngology 1972;81:791.

6. Hoffman H, Darnell L. Laryngeal Cancer. National cancer database annual review of care 1995.

7. Steiner W. Experience in endoscopic laser surgery of malignant tumors of the upper aerodigestive tract. Adv Otorhinolaryngol 1988;39:135-44.

8. Rudert H. $\mathrm{CO}_{2}$ laser treatment of carcinoma of the larynx. Annals of Otology, Rhinology, and Laryngology 1988;67:261-68.

9. Steiner W, Ambrosch P. Endoscopic laser surgery of the upper aerodigestive tract. Stuttgart: Thieme; 2000.

10. Thurnher D, Erovic B, Frommlet F, Brannath E, Ehrenberger K, Jansen B, et al. Challenging a dogma - Surgery yields superior long-term results for $\mathrm{T} 1$ a squamous cell carcinoma of the glottic larynx compared to radiotherapy. European Journal of Surgical Oncology 2008;34:692-98.

11. Werner J, Dunne A, Folz B, Lippert B. Transoral laser microsurgery in carcinomas of the oral cavity, pharynx and larynx. Cancer Control 2002;9(5):379-86.

12. Rodel MW, Steiner W, Muller RM, Kron, M. Endoscopic laser surgery of early glottic cancer: Involvement of the anterior commissure. Head and Neck 2009;31:583-92.

13. Pradhan SA, Pai PS, Neeli SI, D'Cruz A. Transoral Laser Surgery for Early Glottic Cancers. Arch Otolaryngol Head Neck Surg. 2003;129:623-25.

14. Ansarin M, Santoro L, Cattaneo A, Massaro M, Calabrese L, Giugliano G, et al. Laser surgery for early glottic caner: Impact of margin status on local control and organ preservation. Arch Otolaryngol Head Neck Surg 2009;135(4):385-90.

15. Hinni M, Salassa J, Grant D, Pearson B, Hayden R, Martin A, et al. Transoral laser microsurgery for advanced laryngeal cancer. Arch Otolaryngol Head Neck Surg 2007;133(12):1198-204.

16. Forastiere AA, Goepfert H, Maor M, et al. Concurrent chemotherapy and radiotherapy for organ preservation in advanced laryngeal cancer. N Engl J Med 2003;349:2091-98.

17. Ambrosch P, Kron M, Steiner W. Carbon dioxide laser microsurgery for early supraglottic carcinoma. Annals of Otology, Rhinology, Laryngology 1998;107:680-88.

18. Rodrigo J, Suarez C, Silver C, Rinaldo A, Ambrosch P, Fagan J, et al. Transoral laser surgery for supraglottic cancer. Head and Neck 2008;658-66.

19. Vilaseca I, Blanch J, Bernal-Sprekelsen M, Moragas M. $\mathrm{CO}_{2}$ laser surgery: A larynx preservation alternative for selected hypopharyngeal carcinomas. Head and Neck 2004:953-59.

20. Martin A, Jackel M, Christiansen H, Mahmoodzada M, Kron $\mathrm{M}$, et al. Organ preserving transoral laser microsurgery for cancer of the hypopharynx. Laryngoscope 2008;118.

21. Head and Neck Surgery-Otolaryngology. Editors: Bailey BJ, Johnson JT, Newlands SD, Calhoun KH, Deskin RW (4th ed). New York: Lippincott Williams and Wilkins 2006.

22. Eckel H, Volling P, Pototschnig C, Zorowka C, Thumfart W. Transoral laser resection with staged discontinuous neck dissection for oral cavity and oropharynx squamous cell carcinoma. Laryngoscope 1995;105:53-60.

23. Steiner W, Fierek O, Ambrosch P, Hammerich C, Kron M. Transoral laser microsurgery for squamous cell carcinoma of the base of the tongue. Arch Otolaryngol Head Neck Surg. 2003;129(January):36-43.

24. Jaulerry C, Rodriguez J, Brunin F, Mosseri V, Pontvert D, et al. Results of radiation therapy in carcinoma of the base of the tongue. The Curie Institute experience with about 166 cases. Cancer 1991;67:1532-38

25. Mendenhall WM, Stringer SP, Moore GJ, Tannehill SP, Cassisi N J. 97 Squamous cell carcinoma of the base of tongue treated with external-beam radiation therapy: A preferred alternative to surgery. International Journal of Radiation Oncology, Biology, Physics 1999;45(3)Supplement 1:197.

26. Lee HJ, Zelefsky MJ, Kraus DH, Pfister DG, Strong EW, et al. Long-term regional control after radiation therapy and neck dissection for base of tongue carcinoma. International Journal of Radiation Oncology, Biology, Physics 1997;38(5):995-1000.

27. Leemans CR, Rammoban T, Nauta JJP, Snow GB. Discontinuous vs. in-continuity neck dissection in carcinoma of the oral cavity. Arch Otolaryngol Head Neck Surg 1991;117:1003-06.

28. Tesseroli M, Calabrese L, Carvalho AL, Kowalski L, Chiesa F. Discontinuous vs in-continuity neck dissection in carcinoma of the oral cavity. Experience of two oncologic hospitals. Acta Otorhinolaryngol Ital December 2006;26(6):350-55.

29. Holsinger F, Nussenbaum B, Nakayama M, Saraiya S, Sewnaik A, McWhorter A, et al. Current concepts and new horizons in conservation laryngeal sugery: An impotant pat of multidisciplinary care. Head and Neck 2009. ePub 8/11/2009.

30. Steiner W, Vogt P, Ambrosch P, Kron M. Transoral carbon dioxide laser microsurgery for recurrent glottic carcinoma after radiotherapy. Head and Neck 2004;477-84.

31. Grant D, Salassa J, Hinni M, Pearson B, Hayden R, Perry W. Transoral laser microsurgery for recurrent laryngeal and pharyngeal cancer. Otolaryngology-Head and Neck Surgery 2008;138:606-13.

32. Ellies M, Steiner W. Peri- and postoperative complications after laser surgery of tumors of the upper aerodigestive tract. Head and Neck 2007;28:168-72.

33. Bernel-Sprekelsen M, Vilaseca-Gonzalez I, Blanch-Alejandro JL. Predictive values for aspiration after endoscopic laser resections of malignant tumors of the hypopharynx and larynx. Head and Neck February 2004;26(2);103-10.

34. Langmore SE, Schatz K, Olson N. Endoscopic and videofluoroscopic evaluations of swallowing and aspiration. Ann Otol Rhinol Laryngol 1991;100(8):678-81.

35. Aviv JE, Kim T, Sacco RL, et al. FEESST: A new bedside endoscopic test of the motor and sensory components of swallowing. Ann Otol Rhinol Laryngol 1998;107(5 Pt 1):378-87.

36. Logemann J. Evaluation and treatment of swallowing disorders. (2nd ed). Austin, TX: Pro-Ed; 1998.

37. Logemann J. Protocol for swallowing management in patients treated for head and neck cancer. Perspectives on swallowing and swallowing disorders (Dysphagia) June 2006;15:22-26.

38. Hirano M, Kuroiwa Y, Tanaka S, Matsuoka H, Sato K, et al. Dysphagia following varying degrees of resection for oral cancer. Annals of Otology, Rhinology and Laryngology 1992;101:13841.

39. Fujiu M, Logemann J. Effect of a tongue holding maneuver on posterior pharyngeal wall movement during deglutition. American Journal of Speech-Language Pathology 1996;5:23-30. 
40. Shaker R, Easterling C, Kern M, Nitschke T, Massey B, et al. Rehabilitation of swallowing by exercise in tube fed patients with pharyngeal dysphagia secondary to abnormal UES opening. Gastroenterology 2002;122(5):1314-21.

41. Lazarus C, Logemann J, Pauloski B, Rademaker A, Helenowski I, Vonesh E, et al. Effects of radiotherapy with or without chemotherapy on tongue strength in patients with oral cancer. Head and Neck 2007;632-37.

42. Kulbersh BD, Rosenthal EL, McGrew BM, Duncan RD, McColloch NL, et al. Pretreatment, Preoperative Swallowing Exercises May Improve Dysphagia Quality of Life. Laryngoscope June 2006;116:883-86.

43. Pauloski BR. Swallowing function after surgery for oral cavity tumors. Perspectives on Swallowing and Swallowing Disorders (Dysphagia) 2001;10:4-9.

44. Mittal BB, Pauloski BR, Haraf DJ, Pelzer HJ, Argiris A, et al. Swallowing dysfunction-Preventative and rehabilitation strategies in patients with head and neck cancers treated with surgery, radiotherapy, and chemotherapy: A critical review. International Journal of Radiation Oncology, Biology, Physics 2003;57(5):1219-30.

45. Grant GD, Salassa JR, Hinni LM, Pearson WB, Perry CW. Carcinoma of the tongue base treated by transoral laser microsurgery, Part I: Untreated tumors, a prospective analysis of oncologic and functional outcomes. Laryngoscope 2006;116(12):2150-55.
46. Aviv JE, Spitzer J, Cohen M, Ma G, Belafsky P, Close LG. Laryngeal adductor reflex and pharyngeal squeeze as predictors of laryngeal penetration and aspiration. Laryngoscope 2002;112(2):338-41.

47. Sittel C, Eckel HE, Eschenbeurg C. Phonatory results after laser surgery for glottic carcinoma. Otolaryngol Head Neck Surg 1998;119:418-24.

48. Roh JL, Yoon YH. Prevention of anterior glottic stenosis after transoral microresection of glottic lesions involving the anterior commissure with mitomycin C. Laryngoscope Jun 2005;115(6):1055-59.

49. Zeitels SM, Hillman RE, Franco RA, et al. Voice and treatment outcome from phonosurgical management of early glottic cancer. Ann Otol Rhinol Laryngol Suppl Dec 2002;190:3-20.

50. Roh JL, Kim DH and Park CI. Voice, swallowing and quality of life in patients after transoral surgery for supraglottic carcinoma. Journal of Surgical Oncology 2008;98:184-89.

51. Oeken J, Hansen U, Thiel S. Swallowing function after endoscopic resection of supraglottic carcinoma with the carbon dioxide laser. Eur Arch Otolaryngol 2001;258:250-54.

52. Preuss S, Cramer K, Klussman J, Eckel H, Guntinas-Lichius O. Transoral laser surgery for laryngeal cancer: Outcome, complications, prognostic factors in 275 patients. European Journal of Surgical Oncology 2009;35:235-40. 